Methods: Urate in plasma and urine were measured after fructose, glucose, or water was administered to Sprague-Dawley rats at doses of 2.5 or $7.5 \mathrm{~g} / \mathrm{kg}$ at a time. Intestinal closed loops were made using approximately $10 \mathrm{~cm}$ of ileum. Phosphate buffered saline was injected into each intestinal loop. After 4 hours, urate levels in the loops were measured.

Results: Plasma urate level was significantly increased within 15-30 min by oral administration of fructose, without an increase in urinary and intestinal urate excretion, and a decreasing tendency of urate clearance was observed. These actions were evident at a dose of $7.5 \mathrm{~g} / \mathrm{kg}$ body weight. The absence of an increase in urinary and intestinal urate excretion indicated that an increase in urate production did not result in the increase in plasma urate level. In the meantime, a decrease in urate clearance was suggested to cause the increase in plasma urate level.

Conclusions: The present study demonstrated that oral intake of fructose induces an increase in plasma urate level within a short time and that a decrease in renal urate clearance could mainly work for that.

Disclosure of Interest: None declared

DOI: 10.1136/annrheumdis-2018-eular.6366

\section{AB1032 $\quad$ HIGHER BODY FAT IS AN INDEPENDENT RISK FACTOR FOR METABOLIC SYNDROME IN GOUT}

J.-J. Liang ${ }^{1}$, S.-T. Ao ${ }^{2}$, Q.-H. Li ${ }^{1}$, Y.-H. Xu ${ }^{1}$, X.-Y. Du ${ }^{1}$, K.-M. Yang ${ }^{1}$, J.-Z. Lin ${ }^{1}$, Y.Q. Mo' ${ }^{1}$ L. Dai ${ }^{1}{ }^{1}$ Department of Rheumatology, Sun Yat-Sen Memorial Hospital, Sun Yat-Sen University, ${ }^{2}$ Zhongshan School of Medicine, Sun Yat-Sen University, Guangzhou, China

Background: The development of gout is associated with obesity and metabolic syndrome (MS). Obesity is defined by body mass index (BMI). However, BMI has being challenged for the limitation of not distinguishing different comprising tissues of the body. Body composition (BC) has been frequently recommended to assess metabolic status and will eventually replace BMI as a more reliable measure.

Objectives: To investigate the characteristics of $\mathrm{BC}$ in gout patients and their significance with disease characteristics.

Methods: BC was assessed in 180 consecutive gout patients and1,860 control subjects (white-collar employees in Zhangjiang InnoPark of Shanghai) by bioelectric impedance analysis. Overfat was defined by body fat percentage (BF\%) as $\geq 25 \%$ for men and $\geq 35 \%$ for women. Demographic and clinical data as well as comorbid diseases were collected simultaneously. For the significant differences in the proportion of gender and age between two groups, the age- and gendermatched control subjects were randomly selected with the ratio of $1: 1$ for further statistics.

Results: (1)Among 180 gout patients recruited, the mean age was $42.5 \pm 15.5$ years, mean serum uric acid (sUA) was $9.0 \pm 2.4 \mathrm{mg} / \mathrm{dl}$ and $17.2 \%$ of patients presented tophi. The mean BMI was $25.4 \pm 3.5 \mathrm{~kg} / \mathrm{m}^{2}$ with $44.4 \%$ overweight and $25.6 \%$ obesity. The mean BF was $26.2 \% \pm 6.6 \%$ with $50.5 \%$ overfat. (2)Compared with control subjects, gout patients were characterised by higher BMI $(25.4 \pm 3.5$ $\mathrm{kg} / \mathrm{m}^{2}$ vs. $\left.24.3 \pm 3.3 \mathrm{~kg} / \mathrm{m}^{2}\right)$, fat mass $(19.3 \pm 6.9 \mathrm{~kg}$ vs. $16.5 \pm 6.2 \mathrm{~kg})$, trunk fat mass $(10.2 \pm 5.4 \mathrm{~kg}$ vs. $8.4 \pm 3.4 \mathrm{~kg})$, BF $(26.2 \% \pm 6.6 \%$ vs. $22.4 \pm 6.2 \%)$, proportion of overfat ( $50.5 \%$ vs. $27.2 \%)$, but lower lean mass $(53.0 \pm 7.7 \mathrm{~kg}$ vs. $55.6 \pm 7.7 \mathrm{~kg}$, all $\mathrm{p}<0.05)$. (3)Compared with normal fat patients $(n=89)$, gout patients with overfat $(n=91)$ presented higher duration of gout, the count of affected joints, flare times in the past year, family history and presence of tophi (all $p<0.05$, table 1). Overfat gout patients also exhibited higher BMI, more obesity, hyper-low density lipoproteinemia, MS and fatty liver (all $p<0.05)$. (4)There were $9(17.3 \%$ ) overfat gout patients in 53 normal weight patients who presented more hypercholesterolemia ( $55.6 \%$ vs. $13.3 \%$ ), hyper-low density lipoproteinemia ( $55.6 \%$ vs. $17.8 \%$ ) and fatty liver $(77.8 \%$ vs. $35.6 \%)$ and less skeletal muscle mass index (SMMI, $8.3 \pm 0.5 \mathrm{~kg} /$ $\mathrm{m}^{2}$ vs. $9.7 \pm 0.7 \mathrm{~kg} / \mathrm{m}^{2}$ ) than those patients with normal fat and weight (all $p<0.05$ ). Meanwhile, there were 44 (34.9\%) gout patients with normal fat among 126 overweight and obesity patients who had less MS ( $40.9 \%$ vs. $63.4 \%)$ and more SMMI $\left(11.1 \pm 0.9 \mathrm{~kg} / \mathrm{m}^{2}\right.$ vs. $\left.10.6 \pm 1.1 \mathrm{~kg} / \mathrm{m}^{2}\right)$ than overfat patients (both $\left.\mathrm{p}<0.05\right)$. (5)Overfat was a risk factor for MS [OR $3.4(1.8,6.4), p<0.001]$ after adjusted by age and gender.
Abstract AB1032 - Table 1. Comparison of disease characteristics between gout patients in BF subgroups

\begin{tabular}{|c|c|c|c|c|}
\hline Clinical characteristic & $\begin{array}{l}\text { all gout patients } \\
(\mathrm{n}=180)\end{array}$ & $\begin{array}{c}\text { over fat group } \\
(\mathrm{n}=91)\end{array}$ & $\begin{array}{l}\text { normal fat group } \\
\qquad(\mathrm{n}=89)\end{array}$ & $\mathbf{P}$ \\
\hline Male, $\mathrm{n}(\%)$ & $168(93.3)$ & $85(93.4)$ & $83(93.3)$ & 1.0 \\
\hline Age, years & $42.5 \pm 15.5$ & $42.8 \pm 15.5$ & $42.3 \pm 15.6$ & 0.804 \\
\hline Duration of gout, years & $3.0(1.0,8.0)$ & $5.0(2.0,9.0)$ & $3.0(1.0,5.0)$ & 0.005 \\
\hline Count of affecting joints & $3.0(2.0 .6 .0)$ & $4.0(2.0,8.0)$ & $2.0(1.0 .4 .0)$ & $<0.001$ \\
\hline Frequency of flare in the last year & $4.0(2.0,10.0)$ & $5.0(2.0,12.0)$ & $3.0(2.0,6.0)$ & 0.002 \\
\hline Tophi, n (\%) & $31(17.2)$ & $21(23.1)$ & $10(11.2)$ & 0.035 \\
\hline Family history, $n(\%)$ & $63(35.0)$ & $39(42.9)$ & $24(27.0)$ & 0.025 \\
\hline Serum uric acid, mg/dl & $9.0 \div 2.4$ & $9.2+2.2$ & $9.0 \div 2.2$ & 0.616 \\
\hline Hypertension, $\mathbf{n}(\%)$ & $58(32.2)$ & $33(36.3)$ & $25(28.1)$ & 0.267 \\
\hline Diabetes mellitus, n (\%) & $26(14.4)$ & $17(18.7)$ & $9(10.1)$ & 0.137 \\
\hline Dyslipidemia, $\mathbf{n}(\%)$ & $117(65.0)$ & $63(69.2)$ & $54(60.7)$ & 0.274 \\
\hline Hypercholesterolemia, n (\%) & $45(25.0)$ & $28(30.8)$ & $17(19.1)$ & 0.086 \\
\hline Hyper-low density lipoproteinemia, n (\%) & $53(29.4)$ & $34(37.4)$ & $19(21.3)$ & 0.022 \\
\hline BMI, $\mathrm{kg} / \mathrm{m}^{2}$ & $25.4 \pm 3.5$ & $27.2+3.4$ & $23.6+2.4$ & $<0.001$ \\
\hline Obesity, n (\%) & $46(25.6)$ & $42(46.2)$ & $4(4.5)$ & $<0.001$ \\
\hline Body fat, $\%$ & $26.2 \pm 6.6$ & $31,1 \pm 4,3$ & $21.1 \pm 4.3$ & $<0.001$ \\
\hline SMMI, $\mathrm{kg} / \mathrm{m}^{2}$ & $10.4 \pm 1.2$ & $10.4 \pm 1.2$ & $10.4 \pm 1.1$ & 0.981 \\
\hline Metabolic syndrome, n (\%) & $82(45.6)$ & $54(59.3)$ & $28(31.5)$ & $<0.001$ \\
\hline Fatty liver, $n(\%)$ & $99(55.0)$ & $60(65.9)$ & $39(43.8)$ & 0.004 \\
\hline
\end{tabular}

BMI: body mass index; SMMI: skeletal muscle mass index

Conclusions: Our results indicated higher body fat in gout patients which is an independent risk factor for MS.

Acknowledgements: The present study was supported by Guangdong Natural Science Foundation, China (Grant no. 2014 A030310086) to Qian-Hua Li.

Disclosure of Interest: None declared

DOI: 10.1136/annrheumdis-2018-eular.6681

\section{AB1033 1 COMORBIDITIES AND METABOLISM INDEXES IN PATIENTS WITH HUA COMPARING WITH NORMAL URIC ACID: A DATA FROM 33319 PATIENTS BETWEEN 2014 AND 2015}

L. Xu, H. Zhu, Y. Su. Dept of Rheumatology and Immunology, Peking University People's Hospital, Beijing, China

Objectives: To investigate and compare the differences in Comorbidities and metabolism indexes between peoples with high and normal uric acid.

Methods: All the outpatients were enrolled in this cross-sectional study from September 2014 to September 2015 in peking university people's hospital. The patients were divided into high uric acid (HUA) and normal uric acid according to the international definition of HUA. The distribution characteristics of UA level and correlations between UA level and prevalence rate of comorbidities and metabolism indexes, including glutamic-pyruvic transaminase, glutamic-oxalacetic transaminase, serum total cholesterol (TC), triglyceride (TG), low density lipoprotein cholesterol (LDL-C), high density lipoprotein cholesterol (HDL-C), blood glucose, creatinine (CRE), carbonyldiamide, estimated glomerular filtration rate (eGFR) were analysed.

Results: ( ${ }^{1}$ Among 33319 outpatients, with an average age of $56.4,44.4 \%$ were men with a higher uric acid level than women $(368 \pm 113.1$ umol/ vs 300 $\pm 95.5 \mathrm{umol} / \mathrm{L}, \mathrm{p}<0.001)$. In all patients, $25.6 \%$ had HUA and the sex ratio was about $1: 1$, while 50-59 age were most common in men and women with 60-69 age were the most. ${ }^{2}$ Both the levels of uric acid in males and females had a significant relationship with the metabolism indexes above-mentioned. However, the levels of uric acid in females had a positive correlation with age and blood glucose, the levels of uric acid in males were negative correlated with age and bloodglucose. And the uric acid levels in females displayed a higher relationship with carbonyldiamide and eGFR. ${ }^{3}$ The prevalences of common chronic metabolic disorders, were significantly increased in HUA patients than those with norma uric acid, such as hypertension, hypertensive renal disease, hyperlipoidemia, diabetes mellitus (DM), diabetic nephropathy (DN), chronic renal failure. And male patients with HUA had higher prevalence of gout than females $(10.76 \%$ VS $2.58 \%, p<0.001)$. In patients with normal uric acid, the prevalence of DM and DN in males was higher than females. In contrast, the prevalence of DM and DN in males was lower than females in the patients with HUA $(25.7 \%$ VS $57.1 \%$, $\mathrm{p}<0.05 ; 6.52 \%$ VS $41.76 \%, \mathrm{p}<0.001)$

Conclusions: The prevalence of HUA in females was showing a increasing trend and the uric acid level in females had more influence in blood glucose and rena function than males.

Disclosure of Interest: None declared

DOI: 10.1136/annrheumdis-2018-eular.1969 